\title{
Nordiques
}

35 | 2018

Tove Jansson : Par delà les genres. Pratiques

linguistiques dans le Norden du XXIe siècle : Quels

enjeux sociaux?

\section{Figurations de l'art et de l'artiste dans la prose pour adultes de Tove Jansson}

\section{Harri Veivo}

\section{(2) OpenEdition \\ Journals}

\section{Édition électronique}

URL : https://journals.openedition.org/nordiques/806

ISSN : 2777-8479

Éditeur :

Association Norden, Bibliothèque de Caen la mer

\section{Édition imprimée}

Date de publication : 1 mai 2018

Pagination : 31-42

ISBN : 979-1-0959140-1-3

ISSN : $1761-7677$

Référence électronique

Harri Veivo, "Figurations de l'art et de l'artiste dans la prose pour adultes de Tove Jansson », Nordiques [En ligne], 35 | 2018, mis en ligne le 02 février 2021, consulté le 06 octobre 2021. URL: http://journals.openedition.org/nordiques/806 


\section{Figurations de l'art et de l'artiste dans la prose pour adultes de Tove Jansson}

Harri Veivo*

\section{RÉSUMÉ}

Tove Jansson fut un auteur et une artiste majeurs dans un siècle marqué par une critique continue de l'art et de sa fonction dans la société. Bien qu'elle n'ait pas suivi les mouvements d'avant-garde ou modernistes, leur préférant une voie singulière, les nombreux artistes, illustrateurs et autres personnages $d u$ monde des arts qui figurent dans ses récits et romans proposent une réflexion complexe sur le travail créatif, sur l'identité de l'artiste et sur l'art dans la société du XX' siècle. Le sujet est particulièrement intéressant dans la mesure où la conception du travail créatif dans la philosophie janssonienne de l'art est large et couvre tout aussi bien la peinture traditionnelle que la créativité au quotidien.

\section{ABSTRACT}

Tove Jansson was a major writer and a major painter in a century that was marked by constant criticism of art and its function in society. While she did not align her visual art with avant-garde or modernist movements but followed rather her own path, the numerous painters, illustrators and other art-related characters in her short stories and novels propose a complex reflection on creative work, on the identity of the artists and on art in the society of the $20^{\text {th }}$ century. The topic is particularly interesting since the basic conception of creative work in Jansson's philosophy of art is wide, covering both traditional painting and creative activities in the everyday life.

\footnotetext{
* Harri Veivo est professeur au Département d'études nordiques de l'université de Caen Normandie.
} 
Tove Jansson, née en 1914 et morte en 2001, était une artiste et une écrivaine d'un siècle de questionnements. Depuis les manifestes de l'avant-garde qui ont marqué les années 1910 et 1920 jusqu'aux jeux conceptuels et postmodernes des années 1980 et 1990, les artistes et les écrivains ont voulu repenser leur propre rôle et la fonction de leurs œuvres dans la société, redéfinir les conditions sociales, économiques et médiatiques qui déterminent leur travail, et redonner un nouveau statut au public, transformer les spectateurs et les lecteurs passifs en créateurs de sens. Il serait néanmoins vain de chercher dans l'œuvre peinte, dessinée ou écrite de Jansson de grandes proclamations sur le travail créatif ou sur le rôle de l'artiste ou de l'écrivain ; elle n’a pas signé de manifeste, ni participé à des mouvements bien définis qui auraient défendu une théorie particulière de l'art ${ }^{1}$. D'une certaine manière, elle arrive à sa maturité artistique dans une période de transition et de relâche qui a succédé au modernisme des années 1920. Comme l'a montré Tuula Karjalainen dans sa biographie de Jansson, Tove Jansson. Tee työtä ja rakasta (Tove Jansson. Travailler et aimer) $)^{2}$, il existait de nombreuses initiatives d'ouverture vers la modernité artistique européenne dans les milieux artistiques de la Finlande et de la Suède que la jeune Jansson fréquentait dans les années 1930. Les expositions sur l'art français qu'elle a vues à Stockholm et à Helsinki lui ont offert la possibilité de se familiariser avec le postcubisme, le surréalisme et les autres mouvements contemporains. Certains acteurs influents du monde artistique, comme le directeur de l'École des beaux-arts d'Helsinki où elle étudiait à l'époque, restaient cependant hostiles à ces projets novateurs qui n'ont pas réussi à s'imposer dans le champ culturel du pays ${ }^{3}$. La guerre a bien entendu ralenti l'appropriation de ces mouvements en Finlande, imposant une césure dans l'évolution de l'art finlandais. Dans son ouvrage sur les années 1940 et 1950, l'historien de l'art Erik Kruskopf a également noté que les nouveaux discours artistiques des années 1930 ne correspondaient pas au projet personnel de Jansson, à son " désir intérieur de raconter " ("sisäiseen kertojantarpeeseensa "), et que, après la guerre, quand la vie artistique a repris de l'ampleur dans les années 1950 et alors que la modernité devenait la norme, elle n'a pas pu suivre la succession rapide de nouveaux " ismes $»^{4}$. Sa carrière personnelle était en décalage par rapport au dynamisme du champ artistique et littéraire finlandais, lui-même fortement perturbé par la guerre ; on pourrait dire qu'elle est devenue artiste dans une période qui n'était pas artistique.

Mon intention dans cet article est d'aller à l'encontre de ce constat rapide et d'essayer de reconstituer une réflexion sur l'art et l'artiste, et même les rudiments

1 Elle a cependant publié plusieurs essais sur la poétique dans la littérature pour enfants.

2 Tuula Karjalainen, Tove Jansson. Tee työtä ja rakasta, Helsinki, Tammi, 2013.

3 Ibid., p. 31-36.

4 Erik Kruskopf, Valon rakentajat. Suomalaista kuvataidetta 1940-1950-luvuilta, Helsinki, SKS, 2010, p. 37. 
d'une théorie de l'art, dans l'œuvre littéraire de Jansson ${ }^{5}$. Bien qu'elle n'ait pas publié de manifeste et ou d'essai théorique et que mon sujet puisse ainsi sembler inexistant, cet objectif n'est pas sans raison d'être. Les récits et les romans de Jansson proposent au lecteur de nombreux personnages, artistes ou écrivains, et de nombreuses scènes et histoires sur le travail créatif. À partir d'une réflexion sur ces personnages, histoires et scènes, je cherche à comprendre comment ces récits et figures peuvent être analysés, interprétés et complétés pour faire émerger une conception de l'art dans un sens large. Cette conception couvre toute sorte de travail créatif consistant à façonner ou à donner une forme esthétique, dans un sens somme toute assez kantien, mais indépendamment du matériau ou des cadres institutionnels ou économiques qui servent normalement à distinguer les genres artistiques entre eux, d'une part et l'art du divertissement, des hobbies et de la création populaire, d'autre part. Il sera donc question ici des artistes et des écrivains, mais aussi des maquettistes et même d'une petite fille et de sa grandmère, tous engagés dans une activité qui vise à donner forme à ce qui en manque, cette activité s'insérant dans des contextes d'interaction sociale.

Mon approche s'articulera autour de trois sous-thèmes. Je porterai d'abord mon attention sur des récits et des scènes où l'artiste est décrit dans une situation sociale de la vie quotidienne ou bien parmi ses semblables, dans le milieu artistique. Il sera question dans un premier temps du regard porté par ses concitoyens sur l'artiste, mais aussi des modalités d'interaction qui lient l'artiste à la société et à ses collègues. J'aborderai ensuite l'artiste au travail et tenterai de voir comment Jansson décrit les valeurs et les principes qui définissent la création artistique, et, par extension, l'artiste par cette activité créative. Le troisième sousthème concerne le travail créatif, dans un sens plus large qui englobe non pas uniquement l'art, mais aussi la création populaire et l'interaction sociale qu'elle comporte et, par voie de conséquence, le travail de négociation des objectifs, des désirs et des valeurs qui en découle.

\section{L'ARTISTE DANS LA SOCIÉTÉ}

Deux récits publiés par Jansson dans les années 1930 montrent comment elle percevait l'image et la situation sociale de l'art et de l'artiste dans deux milieux différents, à Paris et à Helsinki. Le titre du récit " Quatz'Arts ", paru dans le journal Svenska Pressen en 1938, provient de la fête traditionnelle des étudiants de l'École nationale supérieure des beaux-arts de Paris ${ }^{6}$. Cette fête prenait la forme d'un cortège et d'un bal costumé dans une salle décorée pour l'occasion et jouissait d'une renommée importante dans le folklore étudiant parisien. Fondé sur

5 À l'exclusion, pour des raisons d'espace, de livres sur les Moumines.

6 Republié dans Tove Jansson, Bulevarden och andra texter, S. Happonen (éd.), Helsingfors, Förlaget, 2017, p. 77-86. 
l'expérience personnelle de l'auteur et clairement autobiographique dans son approche, le récit raconte la participation d'une jeune étudiante étrangère à cette fête. Il met en valeur l'esprit de tolérance que la population parisienne manifeste par rapport à la vie de bohème et irrévérencieuse des étudiants ; même les propriétaires des restaurants et la police font preuve d'une certaine souplesse, et la concierge qui ouvre la porte à la jeune femme à son retour est tout heureuse qu'elle ait pu participer à la fête. Cet esprit de tolérance contraste avec le regard porté sur l'artiste dans un autre récit de l'époque, "Hyra rum " ( Louer une chambre »), publié également dans Svenska Pressen en $1939^{7}$. Ce texte raconte les péripéties d'une jeune peintre qui cherche désespérément un appartement à louer à Helsinki. Dans une période de pénurie de logements, les propriétaires sont les maîtres du jeu, et le métier d'artiste peintre, associé inéluctablement au manque d'argent et à un mode de vie non conventionnel, n'est pas un avantage à leurs yeux. Dans le récit, l'identité sociale de la jeune femme est l'objet d'étonnement et de moquerie de la part des autres personnages ; à la fin, la protagoniste cherche à la dissimuler pour décrocher le contrat de location tant convoité ${ }^{8}$.

$\mathrm{Si}$, dans ces deux récits, Paris fait preuve de plus d'ouverture d'esprit qu'Helsinki quant à la place accordée aux arts et artistes au sein de la société, la situation s'inverse si nous regardons l'image que l'œuvre janssonienne offre du milieu des artistes vu de l'intérieur. Le Paris des années 1930 fut une expérience complexe pour Jansson. D’une part, elle savourait la liberté et la richesse de la vie artistique de cette ville qui était toujours la capitale mondiale de la modernité ; mais d'autre part, elle était déçue par l'esprit qui régnait à l'École des beaux-arts, préférant étudier dans les académies libres? . Cette expérience est présente dans «Quatz'Arts » où l'indulgence des Parisiens par rapport à la vie artistique est accompagnée de plusieurs remarques sur l'intolérance - xénophobie, bizutage, mépris, misogynie - qui marque le milieu des étudiants de cette école prestigieuse. Tout autre est l'image que Jansson donne des cercles artistiques d'Helsinki. Dans " Avslutningsdag " ( Le jour de la fin des études ») ou bien « Hippa » ( Une fête » $)^{10}$, deux récits publiés respectivement en 1998 et 1968, le cercle des artistes est égalitaire et tolérant, respectueux des divergences de caractère, d'opinion et d'origine. Il s'agit ici du milieu que l'auteur connaissait personnellement : l'École des beaux-arts d'Helsinki où elle a étudié et le cercle familial des amis artistes de ses parents. La vision positive qu'elle en donne s'explique peut-être ainsi : elle n'a pas eu à justifier

7 Ibid., p. 117-125.

8 Voir l'assimilation des artistes avec les SDF et les Tziganes dans Tove Jansson, «Brev till Konikova ", in Meddelande, Helsingfors, Schildts, 1998, p. 34.

9 Voir Tuula Karjalainen, op. cit., p. 38-39 ; Boel Westin, Tove Jansson. Ord, bild, liv, Helsingfors, Schildts, 1995, p. 93-103.

10 Tove Jansson, "Avslutningsdag ", in Meddelande, op. cit., p. 41-44 ; Tove Jansson, " Hippa », in Bildhuggarens dotter, Stockholm, Bonniers, 2014, p. 33-39. 
son appartenance à ce monde. Le contraste avec Paris est cependant frappant et montre deux visions opposées du champ artistique : dans "Quatz'Arts ", le monde de l'art est valorisé dans la société, mais fortement structuré et hiérarchisé de l'intérieur ; dans les récits sur Helsinki, ce monde est mal vu par la société, mais s'y construit de manière égalitaire. La relation du champ artistique avec l'extérieur produit ainsi deux effets différents de structuration interne.

On peut approfondir cette réflexion sur la dynamique sociale de l'art en analysant l'interaction de l'artiste avec la société, et notamment l'interaction institutionnelle qui est conditionnée par des conventions bien établies et par le statut socioprofessionnel des participants. On en trouve un exemple dans le roman Den ärliga bedragaren (L'honnête tricheuse) publié en $1982^{11}$, une des œuvres les plus énigmatiques de l'auteur. Le protagoniste du roman est une jeune femme qui vit avec son frère sur la côte dans un village de pêcheurs. Pour échapper à sa condition modeste et surtout pour offrir un meilleur avenir à son frère, elle s'introduit dans la vie privée d'une vieille illustratrice de livres pour enfants qui habite dans le même village. Elle devient rapidement tout à fait indispensable à la vieille dame dans la gestion de ses affaires et finit par emménager chez elle, mettant ainsi à exécution son plan initial. Cette opération intéressée et opportuniste produit un effet important sur la vie de l'illustratrice. Elle est habituée à sacrifier son art aux intérêts économiques de sa maison d'édition : excellant dans celui de représenter la nature, elle doit remplir ses scènes magistrales de végétation sylvestre de mignons petits lapins décorés de fleurs, qui sont ensuite accompagnés d'un texte écrit par l'éditeur pour satisfaire au goût du public. En même temps, elle tient à répondre personnellement à chaque courrier des lecteurs. Bien entendu, les contrats qui la lient à la maison d'édition et à d'autres sociétés lui sont très défavorables. Elle est ainsi la figure même de l'artiste qui n'a pas pu défendre son intégrité et celle de son œuvre.

L'intrusion de la jeune femme dans sa vie va changer cette situation : désormais, c'est elle qui répond à sa place aux lecteurs par des lettres type et qui négocie les contrats en sa faveur, faisant fi du désintérêt pour les questions économiques qui a traditionnellement servi à définir l'identité de l'artiste dans le monde moderne. La vieille illustratrice découvre peu à peu sa condition d'artiste exploité et commence à défendre ses intérêts. Dans la scène finale du roman, elle s'assied dans la forêt pour peindre et découvre la terre telle qu'elle est, humide et noire et totalement incompatible avec les petites figures de lapins décorées de fleurs qu'elle dessinait auparavant. La reconquête de l'art et du monde se fait donc au prix de la découverte des relations sociales faussées, mais aussi grâce à une distance rétablie entre l'artiste et son public qui lui garantit son intégrité intellectuelle. Le titre du

11 Tove Jansson, Den ärliga bedragaren, Helsingfors, Schildts, 1982 ; traduction française : L'honnête tricheuse, trad. de M. de Gouvenain, Arles, Actes Sud, 1987. 
roman s'applique donc aux deux femmes, l'artiste étant obligé de tricher dans sa relation avec le public pour défendre la vérité de son travail. L'art est ainsi une recherche de la vérité du monde ; mais comme praxis sociale, elle est indissociable du mensonge qui est nécessaire pour protéger l'artiste et son honnêtetét ${ }^{12}$.

\section{L'ARTISTE ET LA CRÉATION ARTISTIQUE}

Dans ses récits et romans, Jansson ne décrit pas uniquement le contexte social de l'art ; elle réfléchit aussi dans une perspective plus esthétique sur les idées et les valeurs qui interviennent dans le travail créatif. Ces réflexions concernent d'une part, l'œuvre d'art et son mode d'être et d'autre part, la définition de l'artiste et sa condition existentielle telle que déterminée par l'art.

" $[E]$ tt nytt vitt papper, en tom vit yta som varje gång var samma utmaning, samma obegränsade möjligheter och en absolut avskildhet från hjälp utifrån " "Une nouvelle feuille blanche, une surface vierge, chaque fois le même défi, les mêmes possibilités illimitées et un isolement inconditionnel de toute aide extérieure $)^{13}$. C'est par ces mots que le narrateur du récit «Svart-vitt» (« Noir et blanc»), publié en 1972, décrit les conditions de travail d'un illustrateur. Cette citation contient trois éléments essentiels pour comprendre la philosophie de l'art de Jansson et ses conséquences pour l'artiste : les deux idées interconnectées de " défi " et de "possibilité », et l'isolement volontaire et nécessaire de l'artiste engagé dans l'acte créatif. Commençons par le dernier pour remonter vers les deux premiers.

Lidée d'isolement, qui rappelle la discussion sur la relation entre le monde de l'art et la société, se retrouve dans plusieurs récits consacrés aux artistes et écrivains. Dans Stenåkern (Le champ de pierre), roman publié en 1984, le personnage principal se retire du monde pour écrire une biographie d'un homme illustre ${ }^{14}$. Il sera pourtant plus ou moins contraint de passer l'été avec ses filles dans l'archipel, dans un milieu où il ne peut pas éviter le contact avec la société. Dans ces conditions d'isolement mal réussi, le travail créatif devient impossible, au point où le sujet, cet homme riche et illustre dont le protagoniste devrait écrire la vie, se transforme en objet de haine et d'identification dans un cercle complexe qui relie l'obsession à la répulsion. "Robert ", un récit daté de 1991, raconte l'histoire d'un jeune peintre qui a intériorisé la nécessité de s'isoler au point de couper tout lien avec ses collègues et amis. Dans son art, " hans bild fick inte störas av någonting som inte var en organisk del av arbetet, av det absoluta" (" rien ne devait

12 Voir aussi le titre de l'article " Den lömska barnboksförfattaren » (« L'auteur insidieux de littérature pour enfants ») qui propose une réflexion sur la poétique de la littérature pour enfants. Dans Tove Jansson, Bulevarden, op. cit., p. 195-201.

13 Tove Jansson, "Svart-vitt », in Lyssnerskan, Stockholm, Bonniers, 1972, p. 52.

14 Tove Jansson, Stenåkern, Helsingfors, Schildts, 1984 ; traduction française Le champ de pierre, trad. de P. Bouquet et A. Ségol, Nantes, L'Élan, 1991. 
déranger l'image, rien qui n'appartenait d'une manière organique à l'œuvre, à l'absolu ") ${ }^{15}$. Le récit "Svart-vitt ", cité au début du paragraphe, peut servir de troisième exemple. Son personnage principal, un illustrateur, quitte sa femme et son train de vie pour s'isoler dans une maison inhabitée, dans l'objectif de se consacrer entièrement à son travail et d'honorer une commande d'illustrations pour un recueil d'histoires d'horreur. Cet isolement est d'abord un acte pour retrouver sa capacité à créer, mais il déclenche ensuite une réflexion sur le fondement même du travail artistique. Seul dans la maison, le protagoniste découvre la vérité de son travail antérieur : il n'a fait qu'illustrer, c'est-à-dire que ses images n’ont été que des représentations. Désormais, il faudra qu'elles deviennent « ett stycke verklighet " (" un morceau de réalité ») ${ }^{16}$.

L'isolement apparaît ainsi comme une opération nécessaire pour retrouver la vérité de l'acte créatif. Une œuvre d'art doit émerger ou bien naître d'une manière organique, en suivant ses propres lignes d'évolution et en réalisant ses propres possibilités ; rien ne doit perturber ce processus. Face à une feuille vierge, les possibilités sont illimitées ; mais cela ne signifie pas que l'artiste soit un démiurge libre de créer selon sa propre volonté ex nibilo. Dès que l'acte créatif commence, l'œuvre impose ses conditions à l'artiste. L'art est un travail d'exploration de cette dynamique de possibilités et de limites, de la liberté absolue de l'artiste et de sa soumission inconditionnelle à l'œuvre. D'ailleurs, il est intéressant de noter que l'esquisse ou l'ébauche sont présentées par Jansson comme des œuvres plus authentiques dans le sens où elles préservent cette dynamique d'émergence, alors que l'œuvre dévoilée et exposée au regard du public la perd ${ }^{17}$. Le défi du travail de l'artiste ou de l'écrivain est de savoir à quel moment il est arrivé à la fin " naturelle " de ce processus organique, de savoir s'arrêter quand les possibilités ont été découvertes et transformées en réalité.

On pourrait trouver ici des éléments conceptuels que Jansson partagerait avec les artistes et les philosophes qui ont ouvert la voie vers l'abstraction, notamment avec Clement Greenberg. Ce critique d'art américain a théorisé l'évolution de l'art moderne comme un travail de purification qui cherche à éliminer toute convention et toute représentativité inutiles et à réduire la peinture à son essence viable, cette réflexion s'inscrivant dans une continuité avec l'esthétique kantienne ${ }^{18}$. Quelques pionniers de l'abstraction dans les arts finlandais, comme Sam Vanni, figurent effectivement dans le cercle d'amis de Jansson, et elle a ellemême abordé ce discours visuel dans sa peinture, quoique assez tardivement dans

15 Tove Jansson, «Robert », in Meddelande, op. cit., p. 50.

16 Tove Jansson, «Svart-vitt », in Lyssnerskan, op. cit., p. 49.

17 Voir par exemple "Att få en idée ", in Meddelande, op. cit., p. 20-21, et "Brev till Konikova, in Meddelande, op. cit., p. 29-30.

18 Voir Clement Greenberg, Art and Culture. Critical Essays, Boston, Beacon Press, 1961, et « Modernist painting ", 1960, [www.sharecom.ca/greenberg/modernism.html] (consulté le 15 janvier 2018). 
les années 1960. Dans ses récits et romans sur le travail artistique, le sujet - l'élément que l'abstraction cherchait à éliminer - reste cependant une notion essentielle, mais il n'est pas abordé en termes de représentation qui chercherait une relation mimétique entre l'aspect visuel et sa reproduction sur la toile. Il participe plutôt d'une recherche qui vise la vérité existentielle du sujet et de l'artiste. Dans "Brev till Konikova " ("Lettres à Konikova "), un bref récit épistolaire publié en 1998, mais qui tire son origine de la correspondance entre Jansson et son amie Eva Konikoff dans les années 1940, la narratrice "Tove " s'insurge contre " social tendenskonsten " ( l'art social tendanciel [c'est-à-dire politique] ") et lui oppose « l'art pour l'art» (en français dans le texte) exemplifié par Cézanne ${ }^{19}$. L'art pour l'art ne signifie pas abstraction, mais un art qui garde son autonomie par rapport à la politique et qui cherche "den definitiva iakttagelsen" (" la perception cruciale ») qui permettrait à l'artiste d'atteindre non pas seulement l'aspect visuel, mais "Idé» ("l'Idée ») même de son sujet ${ }^{20}$. L'art pour l'art a ainsi une visée qui va au-delà de la surface perceptible et contingente du sujet vers sa réalité profonde. D'autre part, l'accent mis sur l'autonomie signifie que l'artiste ne peut penser à personne d'autre dans son travail et que, par voie de conséquence et paradoxalement par rapport à l'idée cézannienne de l'art, chaque image est, au fond, un autoportrait ${ }^{21}$.

Cela n'implique cependant pas que l'art serait une entreprise solipsiste. Dans le même récit, l'acte créatif est associé à la recherche d'une place dans le monde, rendue difficile par la guerre (le récit se situe vers 1939-1940) ${ }^{22}$. Le rôle accordé au monde rappelle le travail de l'illustrateur dans "Svart-vitt " pour qui les images doivent appartenir à la réalité et non pas être de simples illustrations. On peut comparer ces idées avec le discours tenu par Samuel, un personnage calqué sur le modèle de Sam Vanni dans le récit "Samtal med Samuel » ( Conversations avec Samuel ») publié en $1998^{23}$. Selon Samuel, jeune peintre conceptualisant dans le récit, un artiste doit penser d'une manière "synthétique ", qui cherche l'unité et est orientée vers une connaissance à venir, et qui, dans ce sens, est fondamentalement différente de l'analyse tournée vers le passé et cherchant à établir des distinctions et à démanteler ce qui est organiquement uni. Ces arguments corroborent les propos de la narratrice Tove dans "Brev till Konikova ". Selon elle, un peintre crée avec son cerveau, son cœur et ses tripes ${ }^{24}$. L'acte créatif mobilise ainsi la raison, les émotions et le corps ; la vérité que l'art cherche est l'ensemble

19 Tove Jansson, «Brev till Konikova ", in Meddelande, op. cit., p. 32.

20 Ibid.

21 Pour une interprétation contemporaine de Cézanne dans le contexte finlandais et suédois, voir Göran Schildt, Cézanne, Stockholm, Wahlström \& Widstrand, 1946. Voir Boel Westin, op. cit., p. 294-295 et 300-302.

22 Tove Jansson, "Brev till Konikova ", in Meddelande, op. cit., p. 30.

23 Tove Jansson, "Samtal med Samuel », in Meddelande, op. cit., p. 45-49.

24 Tove Jansson, "Brev till Konikova ", in Meddelande, op. cit., p. 31. 
dynamique de l'expérience qui lie l'artiste au monde à tous ces niveaux. Cette vérité est nécessairement personnelle, mais elle est en même temps réelle et potentiellement partageable, car elle est médiatisée par le sujet et participe du monde interpersonnel de relations, d'interactions, de perceptions et d'expériences.

\section{CRÉER « HORS DE L’ART »}

La troisième facette du travail créatif que je voudrais aborder dans et à travers l'œuvre janssonienne concerne l'activité créative qu'on pourrait considérer située hors des frontières traditionnelles de l'art. Il s'agit du travail créatif qui ne vise pas un produit final qu'on qualifierait d'œuvre d'art, qui ne mobilise pas un savoirfaire acquis dans une institution spécialisée et qui n’est pas situé dans le cadre institutionnel de l'art. Jansson elle-même désignait ce type d'activité avec le verbe dona. Ce mot, qui appartient au registre plutôt familier du suédois (et à l'argot finnois d'Helsinki), est difficilement traduisible. Il signifie approximativement "s'occuper à faire quelque chose ", comme bricoler, réparer ou organiser quelque chose, mais il désigne aussi l'état d'être engagé dans une activité de loisir, ou bien dans une activité sans objectif particulier, qu'on choisit pour passer le temps. Dans son enfance, Jansson utilisait le verbe dona dans son journal pour contourner les expressions trop chargées appartenant au vocabulaire de l'art, comme créer ou être inspiré, lorsqu'elle décrit ses activités créatives qui prennent des formes diverses allant de la peinture à l'écriture, à la couture et à la construction de huttes ${ }^{25}$.

Cette distinction au niveau du vocabulaire est reprise dans les récits de l'auteur. C'est le cas par exemple du récit "Spökskogen » ( La forêt magique ») dans Sommarboken (Livre d'été) où une grand-mère s'amuse à sculpter des figures d'animaux à partir de bois usé dans une forêt mystérieuse de l'île sur laquelle sa famille séjourne pendant les vacances d'été. Lorsque sa petite-fille demande si elle est en train d'organiser un vernissage, elle nie et répond qu'elle joue et que son activité " inte hade med skulptur att göra, skulptur är något helt annat " (" n' [a] rien à voir avec la sculpture et que la sculpture est tout autre chose $")^{26}$. Il est cependant intéressant de noter que, ici et dans de nombreux autres récits, cette activité ludique est caractérisée de la même manière que le travail artistique. Selon le narrateur de "Spökskogen ", les sculptures de la grand-mère restent, comme les véritables œuvres, à l'état d'ébauche, tout en réussissant à exprimer ce que leur auteur souhaite leur faire dire. Dans «Lokomotiv " ( Locomotive »), récit publié en 1978, le narrateur, dessinateur industriel dans une compagnie de chemins de fer, passe ses soirées solitaires à dessiner des locomotives. Il déclare que cette activité est « en lek, en hobby som inte fär förväxlas med ambition" (" un jeu, un hobby qu'il ne faut

25 Boel Westin, op. cit., p. 21.

26 Tove Jansson, Sommarboken, Helsingfors, Schildts, 1972, p. 16 ; Le livre d'un été, trad. de J. Gauffin, Paris, Albin Michel, 1978, p. 17. 
pas confondre avec l'ambition »), mais il dit néanmoins chercher à décrire "kraften, rörelsen, maskinens suveränitet» ( la force, le mouvement et la souveraineté de la machine ") et "tågets idé" ("l'idée de train ") que même le grand peintre anglais Turner n'a pas réussi à capturer ${ }^{27}$. Comme nous l'avons vu tout à l'heure, la notion d'idée apparaît également dans "Brev till Konikova ", où elle sert à légitimer la conception cézanienne de "l'art pour l'art ». Le hobby sans ambition du protagoniste de "Lokomotiv " est donc comparable sous cet angle au travail très sérieux de la jeune peintre Tove dans "Brev " : tous les deux cherchent l'essence du sujet au-delà de sa surface perceptible. Dans « Dockskåpet » ("Armoire à poupées »), le récit éponyme du recueil où "Lokomotiv » fut aussi publié, un tapissier-charpentier qui a récemment pris sa retraite, appelé Alexander, consacre son temps à la construction d'une maison de poupées. Au début, il ne sait pas ce qu'il va faire ; il commence tout simplement par construire trois meubles miniatures, sans objectif particulier. Ensuite, il entame la construction de la maison, sans plan ; " huset skulle fä växa som det ville, organiskt, rum efter rum " (" en laissant la maison grandir comme elle veut, d'une manière organique, chambre par chambre $)^{28}$. "Dockskåpet " propose ainsi la même conception de l'émergence organique de l'œuvre et la même dynamique entre la liberté de création sans objectif et la soumission de l'artiste aux exigences que les récits consacrés à l'activité artistique proprement dite. Qu'il s'agisse d'une peinture, d'une sculpture ou d'une maison de poupées, l'art naît suivant ses propres possibilités et limites, sans objectif ou finalité extérieure; le travail créatif consiste en leur exploration ${ }^{29}$.

Les récits sur dona apportent cependant un nouvel aspect important à la réflexion janssonienne sur l'art. Dans l'œuvre de l'auteur, ce type d'activités créatives comporte une dimension sociale de partage. Si une œuvre d'art doit être cachée pour protéger sa dynamique d'émergence et si le travail de l'artiste est par voie de conséquence solitaire, la création " hors art " est solidement ancrée dans un contexte social d'interaction, soit directement dans le sens où plusieurs agents participent à l'activité, comme c'est le cas dans " Dockskåpet ", soit indirectement dans le sens où la création sert à structurer et à symboliser les relations sociales, comme c'est le cas dans "Lokomotiv » et "Spökskogen ».

Pour Alexander, le protagoniste de " Dockskåpet », la construction de la maison est tout d'abord un moyen de gérer le temps et de chasser l'ennui. Elle devient ensuite un projet partagé entre lui et son compagnon Erik, une sorte d'outil pour la projection d'un avenir commun imaginaire et émancipé des contraintes de la

27 Tove Jansson, Dockskåpet, Helsingfors, Schildts, 1978, p. 40-41, 44 et 49.

28 Ibid., p. 17.

29 Voir la caractérisation de Jansson de sa propre identité professionnelle dans un article sur l'architecture de la maison Moumine : "Tove Jansson - diversearbetare, målare, byggnadsstäderska och sminkös" ("Tove Jansson - femme à tout faire, peintre, nettoyeuse de bâtiment et maquilleuse "), cité dans Sirke Happonen, «Efterord », in Tove Jansson, Bulevarden, op. cit., p. 262. 
vie quotidienne. La construction prend cependant vite une ampleur obsessionnelle qui vide la vie normale du couple de son contenu. La situation commence à dégénérer lorsqu'Alexander fait appel à un ami pour l'aider dans les installations électroniques de la maison, et que celui-ci s'avère être capable de mieux comprendre qu'Erik les défis techniques du projet. S'ensuit une montée de rivalités et de jalousie qui sera résolue à la fin, dans une scène de violence physique où un véritable drame est évité de justesse et les liens du couple d'Alexander et Erik sont réaffirmés. Dans «Lokomotiv », le narrateur, qui est aussi le protagoniste du récit, dévoile peu à peu son activité à une femme rencontrée par hasard et qui devient d'abord sa gouvernante, puis sa compagne. Ici aussi, cette dimension de partage s'avère problématique : la femme, qui ne comprend pas l'importance de l'activité de dessiner les locomotives, est finalement abandonnée par le narrateur. Si ces deux récits peuvent être lus comme des allégories sur la force destructive que l'art et ses capacités illimitées engendrent, dans "Spökskogen » en revanche, le récit ne fait qu'une brève allusion à cette force incontrôlée que l'art peut avoir et propose un dénouement heureux où les deux personnages principaux, la jeune fille Sophie et sa grand-mère, sont unies avec bonheur dans l'activité créative partagée.

\section{Conclusion}

Quelle que soit l'issue de ces récits sur l'activité de dona, il est important de voir, premièrement, qu'ils établissent un parallélisme entre l'art et le travail créatif situé hors du cadre professionnel de l'art et, deuxièmement, qu'ils abordent le travail créatif dans un contexte d'interactions et de relations sociales où le rôle solitaire de l'artiste cède sa place à une capacité à agir partagée. Dans ce sens, dona ressemble à la créativité vernaculaire de la vie quotidienne - le " do-it-yourself» - qui semble prendre aujourd'hui, grâce à l'informatique, une ampleur remarquable ${ }^{30}$. D'autre part, il est possible de voir dans les récits de Jansson des réminiscences de la conception du travail créatif défendue par le mouvement anglais Arts and Crafts de la fin du XIX ${ }^{\mathrm{e}}$ siècle. Ses instigateurs, John Ruskin et William Morris, ont cherché à valoriser la créativité de l'artisanat et l'union organique et authentique entre le travailleur et son travail. Cette conception s'inspire, chez Ruskin surtout, de l'art médiéval. En Suède, elle influença Carl et Karin Larsson et Ellen Key dans leur conception de la maison et de la famille ${ }^{31}$. Bien que des considérations similaires se retrouvent chez Rodin et d'autres figures du

30 Voir à ce propos David Gauntlett, Making is Connecting. The Social Meaning of Creativity, from DIY and Knitting to YouTube and Web 2.0, Cambridge, Polity Press, 2011.

31 Voir par exemple Torsten Gunnarsson, "Carl Larsson, artiste total et fondateur d'un idéal suédois », in Carl Larsson. Limagier de la Suède, Paris, Les Musées de la ville de Paris, 2014, p. 66-76. Rappelons ici que la mère de Tove Jansson, Signe Hammarsten-Jansson, a étudié à Högre Konstindustriella Skolan (École supérieure des arts appliqués) à Stockholm. 
modernisme artistique ${ }^{32}$, le mouvement Arts and Crafts représente une autre couche historique dans la théorisation de l'art par rapport à la réflexion sur l'abstraction, l'art pour l'art, et l'art tendanciel présenté ci-dessus et qui s'ancre solidement dans la modernité du $\mathrm{XX}^{\mathrm{e}}$ siècle.

Il serait ainsi possible de lire dans l'œuvre janssonienne une négociation de deux conceptions de l'art qui sont historiquement et théoriquement distinctes : la première s'inspire de la créativité populaire et la seconde de la recherche moderniste de la vérité de l'art et de son médium. Ces conceptions sont insérées dans un même champ de réflexion par les récits de l'écrivaine. Vues sous ce jour, les figures de l'artiste et les figurations de l'art chez Jansson apparaissent comme un moyen de s'interroger sur les présupposés conceptuels majeurs de notre temps concernant l'activité créative ainsi que sur les conséquences concrètes et sociales qu'ils peuvent engendrer. L'intérêt particulier de cette réflexion réside dans le refus de l'auteur de choisir entre les conceptions qu'elle interroge. Chez Jansson, la modernité existe dans une relation dialogique avec l'histoire; l'art pour l'art cézannien communique avec la création populaire ruskinienne ; le peintre, l'illustrateur et le créateur d'une armoire à poupées sont tous engagés dans une même aventure d'émergence de formes et exposés aux mêmes conflits et tourments dans la vie sociale.

32 Voir Jyrki Siukonen, Vasara ja hiljaisuus. Lyhyt johdatus työkalujen filosofiaan, Helsinki, Kuvataideakatemia, 2011. 